\title{
Les pôles d'excellence rurale : regards sur une politique française en direction de l'espace rural
}

\author{
Christel Alvergne, Université de Bordeaux III \\ Priscilla De Roo, DIACT (ex-DATAR)
}

Les espaces ruraux français ont changé de visage. Ils ont connu depuis vingt ans une véritable « transition démographique » et bénéficient d'un solde migratoire largement positif. Ils sont à présent imbriqués dans une dynamique territoriale globale qui touche en même temps les espaces urbains, périurbains et ruraux. On a pu ainsi parler de « renaissance du rural ». Mais il ne s'agit plus du rural «classique » des années 1960. La mobilité résidentielle hors des villes, la généralisation d'un mode de vie urbain, l'exigence nouvelle pour un environnement et une vie de qualité, la valorisation dans l'imaginaire collectif du patrimoine culturel et naturel transforment profondément la nature de ces espaces et le regard porté sur leur attractivité. Les territoires ruraux offrent non seulement des ressources récréatives et résidentielles, mais également des ressources économiques stratégiques dans une perspective de développement durable.

L'analyse de ces nouvelles dynamiques, qui se différencient largement selon les régions, a donné lieu à une typologie de « trois France rurales » (travail réalisé par la Société d'études géographique et sociologique appliquées [SEGESA] en 2003) :

- les «campagnes des villes », espaces ruraux dont les caractéristiques et les dynamiques sont directement reliées à celles du monde urbain, et qui tirent parti de l'étalement des villes;

- les «campagnes fragiles », espaces en perte de vitesse démographique et économique, souvent isolés géographiquement. Se posent dans ces espaces des enjeux d'accès aux services publics et d'isolement social ${ }^{1}$;

- les «nouvelles campagnes» où apparaissent des dynamiques émergentes. S'y développe une économie résidentielle propre aux espaces "périphériques » devenus attractifs (régions du sud et de l'ouest, régions littorales), motrice d'une nouvelle forme de développement économique local.

Le monde rural se décline donc au pluriel, et cette diversité de situations appelle des politiques publiques différenciées. Certains espaces ruraux en transition sur lesquels pesaient des incertitudes pour l'avenir enregistrent des taux de croissance démographique positifs. On assiste à leur retour en grâce, que les statistiques confirment : $60 \%$ des communes rurales ont vu leur population augmenter entre les deux derniers recensements. Le monde rural ne semble plus être condamné à la désertification du fait de l'attractivité des villes. Les migrations concernent une population au mode de vie urbain et ont des impacts sur la demande de services. Elles débouchent sur une tertiarisation de l'économie rurale, déjà largement engagée et que les Pôles d'excellence rurale (PER) reconnaissent.

Il y a donc là une dynamique qui doit être comprise, accompagnée et soutenue. L'aménagement du territoire a encore une responsabilité sur les espaces ruraux, car il intervient sur l'équilibre général de son organisation. Mais le mode d'action publique doit changer et ne pas se cantonner à une politique traditionnelle de « discrimination positive » accompagnée d'un zonage 
préférentiel. Tous les territoires ont leur chance. La nouvelle approche se fonde sur la conviction selon laquelle les territoires ruraux constituent des réservoirs de croissance et d'excellence au niveau national, et contribuent ainsi à l'attractivité et au développement de la France.

\section{Les pôles d'excellence rurale prennent ainsi acte des mutations sociétales et de leur inscription territoriale.}

Face à ces évolutions, la politique française de développement rural se diversifie et se décline en plusieurs facettes :

- Le maintien d'un traitement particulier pour les espaces fragiles : politique spécifique sur les Zones de revitalisation rurale (ZRR) définies à partir de critères démographiques et économique ${ }^{2}$. Ces zones bénéficient d'aide financière et d'un régime fiscal dérogatoire pour les entreprises qui s'y localisent. Ce zonage couvre environ la moitié de l'espace national, mais concerne de moins en moins d'habitants.

- Un souci de maintien de la présence des services publics, alors que les logiques de rationalisation budgétaire les orientent vers les zones à forte densité : signature entre 1'Association des maires de France et le Premier ministre d'une charte des services publics en milieu rural associant une quinzaine de grands opérateurs de services. Cette charte garantit une concertation locale préalable à toute réorganisation ou fermeture d'écoles, de bureaux de poste, de gares, de tribunaux ou de trésoreries. Une dotation de 50 millions d'euros est prévue et mise à disposition dès 2006 pour le financement de projets alternatifs comme, par exemple, l'amélioration du fonctionnement des «maisons de service public » ou de tout autre mode innovant d'offre de services.

- Une valorisation des ressources résidentielles et économiques avec les Pôles d'excellence rurale lancés par la DIACT (Délégation interministérielle à l'aménagement et à la compétitivité des territoires) (ex Datar) 2006. Ils se veulent être une déclinaison rurale des pôles de compétitivité et s'intégrer dans la stratégie de Lisbonne. Mais ils prennent en compte l'impératif d'équité territoriale en ciblant les espaces ruraux, qui, par hypothèse, ont plus de difficulté au démarrage. Un appel à projets est ainsi lancé autour de la notion d' « excellence rurale ». Il est spécifiquement destiné aux territoires ruraux et exclut les aires urbaines de plus de 30000 habitants. Les projets sont financés par l'État (incluant les fonds européens) à hauteur maximale de $33 \%$ de l'investissement global (et $50 \%$ pour les Zones de revitalisation rurale), dans une limite de un million d'euros. D'autres contributeurs sont appelés à investir, comme les collectivités locales (intercommunalités, départements, régions) ainsi que le secteur privé, avec un accent fortement mis sur le partenariat public-privé (PPP).

Cet appel à projets promeut quatre « filières » économiques, qu'elles relèvent de l'économie productive ou de l'économie résidentielle :

- la culture, le patrimoine entendu au sens large et leur valorisation économique pour favoriser l'attractivité (ce thème a connu un grand succès et a attiré $41 \%$ des projets déposés);

- les services innovants aux ménages nouvellement installés, correspondant aux attentes d'une population se déplaçant avec leur mode de vie urbain (19\% des dossiers se situent dans cet objectif);

- les agro-ressources pour participer au développement durable ( $22 \%$ des projets déposés);

- le secteur des petites entreprises, de l'artisanat et du commerce pour renforcer leur rôle de premier pourvoyeur d'emplois $(18 \%$ des projets déposés).

Les pôles d'excellence rurale prennent ainsi acte des mutations sociétales et de leur inscription territoriale. Ils tentent de les projeter avec une approche qui apprivoise les impératifs de compétitivité dans une tradition française d'équité territoriale. Ainsi, la sélection des projets s'appuie sur un mixage de cinq critères : perspectives de création d'emplois locaux, caractère innovant du produit ou de la filière, inscription dans une logique de développement durable, ancrage rural fort, gouvernance du projet et collaboration entre acteurs. Au total, l'appui du gouvernement au Pôle d'excellence rurale atteindra 235 millions d'euros pour 379 pôles labellisés, les deux tiers des projets étant 
localisés en Zones de revitalisation rurale. Seuls les investissements matériels sont soutenus par l'État.

L'objectif est de créer 35000 emplois dont 13000 emplois directs en deux ans et demi dans les espaces ruraux. Comment s'inscrit cette initiative dans l'édifice politique français et européen ? Peut-on détecter, en filigrane de cet appel à projets, de nouveaux objectifs pour la politique rurale française? Quels liens avec la stratégie de Lisbonne et l'articulation avec Bruxelles ? Nous proposons dans cet article un éclairage sur ces PER à travers deux aspects : 1) renouvellement des dynamiques territoriales et réponses en termes de politiques publiques; 2) insertion et place des PER dans le dispositif actuel de l'aménagement du territoire.

\section{Les PER : quel changement de philosophie politique face à des dynamiques rurales renouvelées?}

Comment peut-on analyser les PER par rapport aux autres politiques menées en direction des territoires ? En quoi les PER constituent-ils une nouveauté ou au contraire un prolongement des politiques en cours dans les espaces urbains, périurbains et ruraux?

\section{Passage de la compétitivité polarisée à l'excellence diffuse}

Cette nouvelle initiative s'inscrit dans les objectifs de compétitivité édictés à Lisbonne qui sont devenus un objectif central pour les politiques territoriales françaises, tout en s'en démarquant profondément. Les « pôles de compétitivité » témoignent en effet de cette volonté de l'État de promouvoir des coopérations productives entre le secteur privé, les centres de recherche et les universités. Il s'agit donc d'exploiter les avantages comparatifs de la proximité territoriale pour mieux mettre en synergie les savoir-faire entrepreneuriaux, techniques et scientifiques nécessaires à l'innovation.

Mais cette volonté, qui tend à promouvoir la polarisation, entre en contradiction avec les objectifs de cohésion territoriale et d'équité spatiale longtemps au cœur de l'aménagement du territoire en France, tout en négligeant les « clientèles » de l'espace rural et sa nouvelle attractivité.

On assiste donc à un glissement sémantique, de la compétitivité vers l'excellence, qui révèle d'autres formes de dynamiques économiques territoriales pas seulement tournées vers l'exportation, mais aussi vers le développement local. On part de l'hypothèse classique du développement local selon laquelle tout territoire dispose d'atouts qu'il convient de valoriser. La confrontation des ressources territoriales au marché et à la concurrence impose ainsi d'identifier des créneaux d'excellence. Un tel objectif ne peut être atteint sans interpeller les acteurs locaux, stimuler l'esprit entrepreneurial et les potentiels de coopération. Or, pour cette alchimie du développement, certains territoires sont moins dotés que d'autres, et en particulier les espaces ruraux.

La logique qui a présidé à la conception des PER est donc double. L'État veut montrer qu'il ne se contente pas de donner le signal de départ de la course à la compétitivité et d'observer passivement le résultat de la mise en concurrence, mais qu'il est attentif à ceux des concurrents qui ont plus de difficulté que les autres. «La solidarité entre territoires ne veut pas dire assistanat. Ce n'est pas subventionner passivement, c'est donner aux territoires qui ont des projets la possibilité de les réaliser. Et aider davantage les projets de ceux qui ont le plus d'obstacles à les franchir. C'est cela la philosophie des pôles d'excellence rurale. $»^{3}$

\section{On assiste donc à un glissement sémantique, de la compétitivité vers l'excellence.}

\section{De l'économie productive à l'économie résidentielle}

L'économie résidentielle (culture, patrimoine, tourisme et services à la population), en particulier dans un pays patrimonial comme la France, constitue l'un des piliers du processus de création de la valeur ajoutée et de l'emploi à l'échelle d'un territoire local. Dans certains espaces, en particulier " périphériques » ou ruraux, mais également dans certaines grandes villes, le poids de cette économie dépasse largement celui de l'économie productive. Cette dynamique se développe en dehors des espaces centraux de la mondialisation, mais constitue, pour un élu local qui a des comptes à rendre à ses administrés, le vrai thermomètre d'un « territoire qui gagne ». Plusieurs des axes de l'appel à projets tendent à valoriser cette économie résidentielle à travers des activités de services à la personne 
et la valorisation des potentiels culturels et touristiques locaux.

À ce jour, le développement d'une économie résidentielle a essentiellement bénéficié aux littoraux. L'objectif de l'appel à projets est donc de favoriser un plus grand équilibre du développement et de l'attractivité en orientant les investissements vers des espaces plus « intérieurs ». De fait, les résultats montrent une concentration des projets financés dans le Massif central, le nord-est de la France en reconversion économique ou la " diagonale des faibles densités » qui court des Ardennes au sud du Massif central ( 7 à 8 pôles en Corrèze, dans le Cantal, l'Ardèche et en Lozère, ainsi que dans les Ardennes et en Moselle, soit le double de la moyenne nationale).

\section{Les acteurs locaux ont souvent regretté que les pays ne permettent pas la mise en œuvre d'actions concrètes qui se traduisent par des investissements.}

\section{L'inscription des PER dans les pratiques de l'action publique d'organisation territoriale}

Au début des années 2000, un nouveau mode d'intervention territoriale est consacré, qui privilégie le bottom up après des années de politiques d'aménagement du territoire centralisé. La coopération entre collectivités locales doit se fonder sur des projets. La coopération de «projet » est facilitée à travers les «pays » en zone rurale, et les «agglomérations » en zone urbaine. Les PER s'inscrivent-ils dans cette logique? Constituent-ils un outil adapté aux recompositions territoriales actuelles?

Dans les PER, la plupart des projets sont portés par des communautés de communes (200) et des pays (90). Ils s'inscrivent donc parfaitement dans la logique de territoires de projets telle qu'elle est mise en place depuis maintenant presque dix ans. Les projets retenus doivent associer partenaires publics et privés (mais sans obligation de créer une forme juridique pour concrétiser ce partenariat, à la différence des pôles de compétitivité).

L'appel à projets PER ne constitue pas une nouveauté sur le plan de la démarche, car la procédure de l'appel à projets et la mise en concurrence des territoires par l'État central est devenu un nouveau mode de «gouvernement à distance $»^{5}$ permettant de contourner le transfert de compétences induit par la décentralisation. D'autres initiatives l'ont précédé (GAL Leader, appels à projet de la DATAR pour les pôles de compétitivité, les coopérations métropolitaines, les villes moyennes).

Mais, avec cet appel à projets, on passe clairement d'une aide de l'État central en matière d'ingénierie à une aide en matière d'investissement, et on privilégie donc clairement l'entrée «projet» et investissements par rapport à l'entrée « territoire » et organisation.

Il n'en demeure pas moins que plusieurs questions se posent quant aux transformations de l'action publique sur le territoire.

\section{Concurrence entre « pays » et PER ?}

La première question concerne le périmètre territorial « pertinent » de l'action en direction du monde rural. La mise en œuvre des «pays » par la loi Pasqua ${ }^{4}$ de 1995, puis sa confirmation par la loi Voynet de 1999, a consacré une action publique déjà fléchée sur les territoires ruraux. Elle était basée sur la notion de « territoire de projet». Cette notion de projet donnait la primauté à la mobilisation des acteurs locaux, à leur capacité de coopération sur des périmètres larges transcendant la coupure entre rural et urbain, bref elle donnait une prime à la gouvernance locale. Elle s'est donc traduite concrètement par l'octroi de crédits d'ingénierie favorisant cette synergie. Les pays résultent d'une approche assez « soft » de l'intervention de l'État sur l'organisation des territoires ruraux. D'ailleurs, les périmètres de coopération qui dessinent un pays ne se traduisent pas par une structure d'intercommunalité constituée juridiquement, mais par la mise en place souple d'un syndicat mixte ou d'un groupement d'intérêt public.

Les acteurs locaux, et les élus principalement, ont souvent regretté que les pays, territoires de projet, ne permettent pas la mise en œuvre de projets au pluriel, c'est-à-dire, d'actions concrètes qui se traduisent par des investissements. Les PER veulent délibérément répondre à cette critique. Ils veulent quitter la méthodologie de l'organisation pour passer à l'action. On a ainsi pu estimer que les PER traduisaient une volonté politique de mettre en cause les pays, décidés par des 
gouvernements de la majorité précédente. Dans les faits, ce risque a été largement évité, car non seulement les pays sont éligibles aux PER, mais aussi, beaucoup de projets de PER ont permis de mettre en réalisation des actions découlant de la réflexion précédente sur la stratégie territoriale à l'échelle du pays. Enfin, les PER, puisqu'ils mettent en place des investissements, doivent clairement être portés par des maîtres d'ouvrage dont les compétences sont définies par la loi. De ce fait, les projets ont permis de renforcer l'intercommunalité rurale (communautés de communes) et de lui donner un souffle politique nouveau par le simple fait de lui apporter des munitions financières.

\section{Le partenariat public-privé dans les PER}

Plusieurs projets comptent développer le partenariat public-privé. Ainsi, le pôle de plantes médicinales de Saint-Pouçain-sur-Slouve (Allier) associe le Laboratoire de phytosanitaire et d'herboristerie (LPH) à l'université de Clermont-Ferrand. Dans le domaine touristique, l'investissement du groupe hôtelier Pierre et Vacances en Sologne s'appuie sur une collectivité locale pour créer des pistes cyclables alentour et un complexe aquatique. L'association de grands opérateurs du secteur touristique comme Pierre et Vacances avec des collectivités locales est un signal important des opportunités offertes par le développement rural dans une approche partenariale entre public et privé.

\section{Une recentralisation de l'action sur le local ?}

La seconde question porte sur les modalités de l'intervention territoriale de l'État dans un contexte de décentralisation. On peut dire que l'appel à projets PER illustre parfaitement le nouveau mode de fonctionnement de l'État central fait de maintien du pouvoir de décision par la mise en distance de certains acteurs. Et ceci, à la lumière de plusieurs constats :

- La procédure d'appel à projets met en concurrence les territoires directement et contourne de ce fait les stratégies des acteurs « intermédiaires » comme les régions (conseils régionaux).

- La logique de l'État central est clairement allocative (on alloue un label PER et une enveloppe de crédits d'investissements) et non plus seulement redistributive (sur la base d'une carte des territoires prioritaires de la redistribution). On peut considérer que l'État se spécialise dans cette logique allocative, vue idéologiquement comme positive, alors qu'il confine les collectivités locales à la logique redistributive, qui est considérée comme moins valorisante.

\section{La procédure d'appel à projets met en concurrence les territoires directement et contourne de ce fait les stratégies des acteurs « intermédiaires » comme les régions (conseils régionaux).}

- Le temps de l'appel à projets a été si court que la concertation avec les grandes fédérations d'élus n'a pas pu se faire. Ainsi, la cohérence entre les stratégies des régions et celle des territoires locaux n'a pas eu lieu. Sur ce point, deux conceptions s'affrontent. La première conception, «modernisatrice $»$, considère que les niveaux pertinents de l'action territoriale sont la région et l'intercommunalité (dans les villes, s'entend). Cette position est encore largement portée par la DIACT à travers notamment les contrats de plan État-région et la régionalisation des programmes opérationnels européens. Une seconde conception défend la proximité du terrain et privilégie le passage par le département pour atteindre des objectifs sur l'espace rural. Les PER ont été ainsi suivis par les préfets de département qui ont réalisé localement leur expertise en vue de la sélection. Mais il s'agit là d'une implication des services déconcentrés de l'État dans une logique de proximité et non d'une concertation avec les collectivités. Ce manque de cohérence avec les stratégies des collectivités locales a été fortement mis en cause, d'autant plus que celles-ci amènent le complément de deux tiers des fonds nécessaires à la réalisation des projets.

- Le mode de gestion des crédits alloués au 379 PER est particulier et conforte la mise à distance de l'État. D'abord, en amont, une ligne unique regroupe les crédits des différents ministères concernés par les PER (aménagement du territoire, agriculture, industrie, écologie). Ces crédits sont versés sur le fonds interministériel que gère la DIACT. Mais, en aval, les crédits de paiement sont également gérés par une structure unique qui, pour des raisons d' « efficience », n'est pas située au cœur de l'État, 
mais prend la forme d'une agence jouant le rôle de prestataire extérieur. En cela, les PER participent à l' "externalisation» de certaines fonctions de l'État (gestion), comme cela a été déjà fait avec l'Agence nationale pour la rénovation urbaine dans le cadre de la politique des quartiers urbains sensibles.

- Les projets correspondent plus à un redéploiement de crédits de droit commun qu'à l'ouverture de nouveaux crédits pour de nouveaux projets. Le temps de l'appel d'offres n'a pas toujours permis l'innovation, il assure par contre le recyclage de projets dormants. Les critères de sélection, la définition floue de la notion d'excellence, le faible taux de rejet des candidatures n'ont pas facilité l'émergence de projets novateurs et ont favorisé dans certains cas un effet d'aubaine. Dans d'autres cas, les collectivités porteuses n'ont pas ressenti la nécessité de financements supplémentaires, mais sont allées à la recherche du label, pour leur permettre d'être « visibles et dans la course ». L'octroi d'un label reconnaissant des réalisations de qualité est donc à dissocier des crédits d'investissements pour le montage de projets pouvant aboutir effectivement à un label. Pour le moment, les PER sont financés « sur plans » sans aucune garantie de réalisation. Enfin, peu de PER mettent en mouvement le partenariat public-privé, et celui-ci est souvent cantonné à la gouvernance sans effet sur les financements.

Pour autant, le succès des PER n'en a pas moins été indéniable. Plus de 700 candidatures ont été déposées. Ils valorisent le lien entre excellence et proximité, ils disent « les petits ruisseaux font les grandes rivières » de la compétitivité. Ils sortent l'espace rural d'une approche compassionnelle ou méthodologique pour les faire entrer de plein pied dans la nouvelle modernité sociétale et économique en prenant en compte leur nouvelle attractivité et leur effet de levier pour le développement durable.
Les PER constituent donc bien une nouveauté remarquable dans l'histoire des politiques françaises en direction de l'espace rural. Non seulement ils répondent à une révolution silencieuse du monde rural, mais ils constituent une sédimentation des modes opératoires de l'État sur le territoire en France.

\section{Notes et références}

1 Dans ces espaces, le vote « non » au référendum est particulièrement élevé, représentant entre $60 \%$ et $70 \%$ des voix.

2 Périmètre des ZRR : les ZRR sont des zones à fiscalité propre dont le périmètre doit obéir aux conditions suivantes :

- la commune est membre d'un établissement public de coopération intercommunale à fiscalité propre;

- la densité démographique de l'arrondissement ou du canton n'excède pas 5 habitants au $\mathrm{km}^{2}$ (ZRR à très faible densité);

- ou la densité démographique de l'arrondissement n'excède pas 33 habitants au $\mathrm{km}^{2}$ (ZRR à faible densité);

- ou la densité démographique du canton ou du territoire recouvert par l'EPCI n'excède pas 31 habitants au $\mathrm{km}^{2}$ (ZRR à faible densité);

- au moins un des critères socio-économiques suivants est satisfait :

- déclin de la population;

- déclin de la population active;

- forte proportion d'emplois agricoles.

3 Discours prononcé à Mende par le ministre de l'intérieur et de l'aménagement du territoire - actuellement Président de la République - le 17 octobre 2006.

4 Note de l'éditeur : En 1995, la loi Pasqua introduisait dans la législation la notion de pays et invitait les territoires à l'expérimentation. La loi Voynet en 1999 consacrait les pays comme des espaces de fédération des acteurs publics et privés autour d'un projet et d'un contrat (source : Projet de territoire.com : http://www.projetdeterritoire.com/spip/ dossier.php3?id_rubrique=73).

5 Epstein, R. (2005). « Gouverner à distance », Esprit, novembre. 